\section{Car Detection Using Cascade Classifier on Embedded Platform}

By: Zulkhairi, MA (Zulkhairi, Muhammad Asyraf) ${ }^{[1]}$; Mustafah, YM (Mustafah, Yasir Mohd) ${ }^{[1]}$; Abidin, ZZ (Abidin, Zulkifli Zainal) $^{[1]}$; Zaki, HFM (Zaki, Hasan Firdaus Mohd) ${ }^{[1]}$; Rahman, HA (Rahman, Hasbullah Abdul) ${ }^{[2]}$

\section{7TH INTERNATIONAL CONFERENCE ON MECHATRONICS ENGINEERING (ICOM)}

Book Group Author(s): IEEE

Pages: $99-101$

Published: 2019

Document Type: Proceedings Paper

\section{Conference}

Conference: 7th IEEE International Conference on Mechatronics Engineering (ICOM)

Location: Putrajaya, MALAYSIA

Date: OCT 30-31, 2019

Sponsor(s): Int Islam Univ Malaysia; Univ Tun Hussein Malaysia; IEEE Robot \& Automat Soc; Inspilogix; ProStrain Technologies; IEEE

\section{Abstract}

Advanced Driver- Assistance Systems (ADAS) help reducing traffic accidents caused by distracted driving. One of the features of ADAS is Forward Collision Warning System (FCWS). In FCWS, car detection is a crucial step. This paper explains about car detection system using cascade classifier running on embedded platform. The embedded platform used is NXP SBC-S32V234 evaluation board with 64-bit Quad ARM Cortex-A53. The system algorithm is developed in $\mathrm{C}++^{+}$programming language and used open source computer vision library, OpenCV. For car detection process, object detection by cascade classifier method is used. We trained the cascade detector using positive and negative instances mostly from our self-collected Malaysian road dataset. The tested car detection system gives about 88.3 percent detection accuracy with images of 340 by 135 resolution (after cropped and resized). When running on the embedded platform, it managed to get average 13 frames per second with video file input and average 15 frames per second with camera input.

\section{Keywords}

Author Keywords: Cascade Classifiers; Object Detection; image processing; Vision-based ADAS; FCW system KeyWords Plus: VEHICLE DETECTION

\section{Author Information}

Reprint Address:

International Islamic University Malaysia Int Islamic Univ Malaysia, Kulliyah Engn, Ctr Unmanned Technol CUTe, IIUM, Gombak, Selangor, Malaysia.

Corresponding Address: Zulkhairi, MA (corresponding author)

+ Int Islamic Univ Malaysia, Kulliyah Engn, Ctr Unmanned Technol CUTe, IIUM, Gombak, Selangor, Malaysia. Addresses:

+ [1] Int Islamic Univ Malaysia, Kulliyah Engn, Ctr Unmanned Technol CUTe, IIUM, Gombak, Selangor, Malaysia

[2] Delloyd R\&D M Sdn Bhd, Klang, Selangor, Malaysia

E-mail Addresses: asyrafzulkhairi037@gmail.com; yasir@iium.edu.my; zzulkifli@iium.edu.my;

hasanzaki@iium.edu.my; hasbullah@delloyd.com

Funding

\begin{tabular}{|l|l|}
\hline Funding Agency & Grant Number \\
\hline International Islamic University Malaysia (IIUM) & \\
\hline Collaborative Research in Engineering, Science and Technology (CREST) & \\
\hline Delloyd RD (M) Sdn. Bhd. & \\
\hline IIUM Research Acculturation Grant Scheme (IRAGS) & P11C2-17 \\
& SP17-029-0291 \\
\hline
\end{tabular}

View funding text

\section{Citation Network}

In Web of Science Core Collection

\section{0}

Times Cited

Create Citation Alert

\section{0}

Cited References

View Related Records

\section{Use in Web of Science}

Web of Science Usage Count

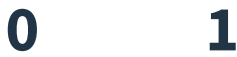

Last 180 Days $\quad$ Since 2013

Learn more

This record is from

Web of Science Core Collection

- Conference Proceedings Citation IndexScience

\section{Suggest a correction}

If you would like to improve the quality of the data in this record, please suggest a correction. 


\section{Publisher}

IEEE, 345E 47TH ST, NEW YORK, NY 10017 USA

\section{Categories / Classification}

Research Areas: Automation \& Control Systems; Engineering

Web of Science Categories: Automation \& Control Systems; Engineering, Electrical \& Electronic; Engineering,

Mechanical

See more data fields

\section{Cited References: 10}

1. Vehicle Detection for Autonomous Parking using a Soft-Cascade AdaBoost Classifier

By: Broggi, Alberto; Cardarelli, Elena; Cattani, Stefano; et al.

2014 IEEE INTELLIGENT VEHICLES SYMPOSIUM PROCEEDINGS Book Series: IEEE Intelligent Vehicles Symposium Pages: 918-923

Published: 2014

2. A Unified Multi-scale Deep Convolutional Neural Network for Fast Object Detection

Times Cited: 497

By: Cai, Zhaowei; Fan, Quanfu; Feris, Rogerio S.; et al.

COMPUTER VISION - ECCV 2016, PTIV Book Series: Lecture Notes in Computer Science Volume: 9908 Pages: 354-370 Published: 2016

3. (r-cnn) Rich feature hierarchies for accurate object detection and semantic segmentation

By: Girshick, R.; Donahue, J.; Darrell, T.; et al.

2014 IEEE C COMP VIS Pages: 2-9 Published: 2012

[Show additional data]

4. Title: [not available]

By: Howard, A. G.; Zhu, M.; Chen, B.; et al.

Times Cited: 211

MobileNets: Efficient Convolutional Neural Networks for Mobile Vision Applications Published: 2017

[Show additional data]

5. Comparison of Forward Vehicle Detection Using Haar-like features and Histograms of Oriented Gradients (HOG)

Times Cited: 1

Technique for Feature Extraction in Cascade Classifier

By: Ibrahim, M. M.; Ali, N. M.

J. Telecommun. Electron. Comput. Eng. Volume: 9 Issue: 2 Pages: 101-105 Published: 2017

N. S. A

6. Vision-based vehicle detection for a driver assistance system

Times Cited: 43

By: Kuo, Ying-Che; Pai, Neng-Sheng; Li, Yen-Feng

COMPUTERS \& MATHEMATICS WITH APPLICATIONS Volume: 61 Issue: 8 Special Issue: SI Pages: 2096-2100 Published: APR 2011

7. Fast Multiclass Vehicle Detection on Aerial Images

Times Cited: 117

By: Liu, Kang; Mattyus, Gellert

IEEE GEOSCIENCE AND REMOTE SENSING LETTERS Volume: 12 Issue: 9 Pages: 1938-1942 Published: SEP 2015

8. Object detection using Haar-cascade Classifier

Times Cited: 1

By: Soo, $S$.

Inst. Comput. Sci. Univ. Tartu Pages: 1-12 Published: 2015

9. Title: [not available]

Times Cited: 1

Group Author(s): Tzutalin

GitHub-tzutalin/labellmg: Labellmg is a graphical image annotation tool and label object bounding boxes in images

Online Accessed:29-Jun-2019

10. Title: [not available]

By: Viola, P.; Jones, M

Times Cited: 1

MERL-A MITSUBISHI ELECTRIC RESEARCH LABORATORY Rapid Object Detection Using a Boosted Cascade of Simple Features Rapid Object

Detection using a Boosted Cascade of Simple Features Published: 2004

\section{Showing 10 of $10 \quad$ View All in Cited References page}

